amended in the light of a more detailed study of the development of the anode-fall region of the discharge. A pseudo-FDS may sometimes develop, leading to misleading results.

(4) The minimum distance from the cathode at which the anode fall develops is strongly dependent on the dimensions of a hollow cathode as well as on the pressure and current density.

(5) A distinction must be made between the behavior of $\mathrm{HC}$ discharges in which the current density may be referred to as either normal or abnormal (by comparison with a plane-cathode discharge).

An analysis of some of these results has led to the following conclusions:

(6) The dependence of the anode-fall development on $x_{c}$ may be simply accounted for in terms of variations in the electron concentration and the random and directed velocities of the electrons.
(7) By relating the magnitude of the enhancement of the discharge efficiency by the HCE to the maximum relative decrease in tube voltage with respect to a planecathode discharge for the same discharge current, it is found that

(a) this enhancement is much smaller than previously believed;

(b) it does not vary widely for any of the gases tested;

(c) it decreases with decreasing current density, approaching zero at "normal" current densities.

(8) The lowest observed cathode fall for a hollowcathode discharge is not appreciably (if at all) lower than the normal cathode fall for a plane-cathode discharge.

Finally, as a consequence of this study of the discharge phenomena, some recommendations for the design of high-efficiency hollow-cathode discharges have been given.

\title{
Pure Space-Charge-Limited Electron Current in Silicon
}

\author{
S. Denda* and M-A. Nicolet \\ Califormia Instiule of Technology, Pasadena, California \\ (Received 29 October 1965 ; in final form 27 December 1965)
}

\begin{abstract}
Phosphorus diffusion on $\pi$-type silicon is used to fabricate $n^{+} \pi n^{+}$structures of base width between $3 \mu$ and $60 \mu$ with $\pi$-type resistivities of $300 \Omega \cdot \mathrm{cm}$ and $8 \mathrm{k \Omega} \cdot \mathrm{cm}$. The $V-I$ characteristics of the structures are measured at room temperature and at liquid-nitrogen temperature. The change in current for constant applied voltage is also observed in that temperature range.

The results are interpreted in terms of simple models based on the assumption that pure space-chargelimited current of electrons is present. The models describe well the characteristics measured on $300-\Omega \cdot \mathrm{cm}$ samples, except for the range of small biases on the thinnest samples. It is concluded that the drift velocity of electrons at $78^{\circ} \mathrm{K}$ tends towards saturation at $1.0 \times 10^{7} \mathrm{~cm} / \mathrm{sec} \pm 10 \%$. The current observed at this temperature actually reaches this value. The critical electric field at $78^{\circ} \mathrm{K}$ is $10^{3} \mathrm{~V} / \mathrm{cm} \pm 30 \%$ but the meaning of this concept for electrons in silicon is vague. The temperature dependence of the current at fixed bias voltages is in general agreement with the variation of the low field mobility. Results obtained on $8-\mathrm{k} \Omega \cdot \mathrm{cm}$ samples need clarification. Effects of breakdown and trapping are not observed.
\end{abstract}

\section{INTRODUCTION}

$T$ $\mathrm{HE}$ credit for giving the first simple theoretical description of unipolar space-charge-limited current (sclc) in a solid is traditionally attributed to Mott and Gurney, ${ }^{1}$ though the roots of the problem may be traced to earlier workers. ${ }^{2}$ According to Mott and Gurney's model, sclc depends linearly upon the static dielectric constant $\epsilon$ of the solid, the charge carrier mobility $\mu$, and the square of the applied voltage $V$.

\footnotetext{
* Permanent address: Electrotechnical Laboratory, Tanashi Annex, Tokyo, Japan.

${ }^{1}$ N. F. Mott and R. W. Gurney, Llectronic Processes in Ionic Crystals (Oxford University Press, Oxford, 1940), 2nd ed. (1957), p. 172.

${ }^{2}$ G. T. Wright, Proc. IEEE 51, 1642 (1963).
}

But these authors also state that the observation of sclc may be impaired because in any real crystal there exist traps where, at sufficiently low temperatures, charge carriers find lower energy levels than in the bands and can be held in a fixed position.

It is only in recent years that the subject has attracted wider attention, motivated partly by the promises which the phenomenon has been claimed to hold in device applications. Pioneered largely by authors such as Rose $^{3}$ and Lampert, ${ }^{4}$ unipolar sclc is recognized today as an efficient tool in the study of the electronic properties of a large variety of solids ranging from

\footnotetext{
${ }^{3} \mathrm{~A}$. Rose, Concepts in Photoconductivily and Allied Problems (John Wiley \& Sons, Inc., New York, 1963).

${ }^{4}$ M. A. Lampert, Rept. Progr. Phys. 27, 329 (1964).
} 
elemental semiconductors to organic crystals. The general picture which has emerged as a result of these efforts is that as a rule-and as foreseen by Mott and Gurney-unipolar sclc is dominated by the presence of charge carrier traps. A large variety of effects can be caused by various combinations of the parameters determing the properties and the distribution of the traps in the solid. As a result, pure (that is, trap-free) sclc has not been observed often. Its properties are known by inference and by theoretical deduction more than by direct experimental evidence. The present study has been undertaken with the aim of providing more complete and direct experimental facts on pure sclc in a solid.

The physical nature and identity of many of the charge carrier traps observed so far in solids is unk nown. An obvious guess is to link them with imperfections. To find a solid in which pure sclc can be observed, one should therefore lay emphasis on extreme perfection. This points towards the choice of an elemental single crystal of which germanium and silicon are presently the best ones.

Germanium has been selected by Dacey and by Shumka ${ }^{6}$ in a similar attempt to investigate the properties of sclc. Dacey's results indicate that the simple theory of Mott and Gurney has to be modified to take into account the field dependence of the charge carrier mobility. He developed an improved theory assuming that the charge carrier mobility varies with the electric field as $E^{-\frac{1}{2}}$. The verification was later provided by Shumka whose work is the first detailed experimental analysis of pure sclc in a solid, using holes as charge carriers. He finds good agreement with Dacey's theory. But to some extent, this must be fortuitous, for it is known that the drift velocity of holes in germanium saturates at high field strengths. One therefore expects that, in extending Shumka's observations to still higher current densities, velocity saturation should be observed when breakdown effects can be avoided.

Silicon, on the other hand, has not been studied as thoroughly. A few authors have examined the injecting properties of contacts in the depletion region of a reverse-biased $p n$ junction and have explained their results in terms of pure sclc. ${ }^{7,8}$ There has even been an attempt to construct a device on this basis. ${ }^{9}$ But the complicated geometry of such structures and their complex field distributions are serious obstacles and impair their usefulness. Recently, Gregory and Jordan ${ }^{10}$ investigated the flow of sclc of holes in silicon at $4.2^{\circ} \mathrm{K}$. Due to the compensated nature of the crystal, trapping

G. C. Dacey, Phys. Rev. 90, 759 (1953).

${ }^{6} \mathrm{~A}$. Schumka and M-A. Nicolet, Procedings of the $7 \mathrm{th}$ International Conference on the Plissics of Semiconductors (Dunod Cie., Paris, 1964), p. 621.

7 J. M. Lavine, Solid-State Electron. 1, 107 (1960).

${ }^{8}$ P. G. Sedlewic, R. E. Onley, and C. R. Kannewurf, SolidState Electron. 7, 225 (1964).

${ }^{3}$ H. Statz and R. A. Pucel, Proc. IEEE 45, 317 (1957).

${ }^{10}$ B. L. Gregory and A. G. Jordan, Phys. Rev. 134, A1378 (1964). effects were found to dominate at these low temperatures and pure sclc was obtained as a transient only. As in germanium, it was found necessary to account for the field dependence of the charge carrier velocity. According to these results, the decrease of the hole mobility is approximately proportional to $E^{-0.6}$. These same authors have also found evidence recently that at $300^{\circ} \mathrm{K}$ trapping effects are absent. ${ }^{11}$

Because of the lesser attention that silicon has received so far with regard to unipolar sclc, it has been selected here as the host lattice for the experiments described below. This choice is further suggested by the dominant position which this material has in the field of solid-state devices, and by the fact that well-established and simple techniques are available for the fabrication of samples. Silicon also has smaller ionization rates than germanium for both electrons and holes. ${ }^{12}$ This moves the limit of avalanche breakdown towards higher voltages and increases the range of voltage and current levels accessible to the experiment.

\section{DEVICE STRUCTURE}

The major problem to solve when unipolar sclc is to flow through a solid is that of finding both a suitably injecting as well as a suitably blocking contact. In the case of a semiconductor such as silicon, $p n$ junctions offer themselves as an attractive solution. Their operation is well understood and they are relatively simple to make. The disadvantage lies in the use of silicon itself, in which charge carriers are present in nonnegligible amounts even at liquid-nitrogen temperature. To minimize this effect it is advantageous to select crystals of low residual doping. Single crystals of silicon of the highest purities available today are always $\pi$ type (slightly $p$ type). Three distinct types of structures with two junctions do exist with a $p$-type base material. These are the $p^{+} p p^{+}, p^{+} p n$ and $n p n$ structures. With an applied bias, four different modes of operation can be distinguished. In the $p^{+} p n$ structure, however, either both contacts inject charge carriers and the current is bipolar ${ }^{13}$ or neither of the contacts injects and only little current flows. There thus remain just two junction devices with which to examine unipolar sclc, the $p^{+} p p^{+}$ and the $n p n$ structure. Their main difference is that the current is carried by holes in the $p^{+} p p^{+}$structure and by electrons in the $n p n$ structure. Both possibilities have been used successfully: the former by Gregory and Jordan and the latter by Dacey and by Shumka in a complementary version of a $p^{+} \nu p^{+}$structure. Since sclc of electrons has never been studied in silicon and because $n$ layers can readily be obtained on $\pi$-type silicon by solid-state diffusion of phosphorus, the $n p n$ structure

\footnotetext{
"11 B. L. Gregory and A. G. Jordan, J. Appl. Phys. 35, 3046 (1964)

${ }_{12}$ J. L. Moll, Physics of Semiconductors (McGraw-Hill Book Co., Inc., New York, 1964), p. 222.

${ }^{13}$ J. W. Mayer, R. Baron, and O. J. Marsh, Phys. Rev. 137, A286 (1965).
} 


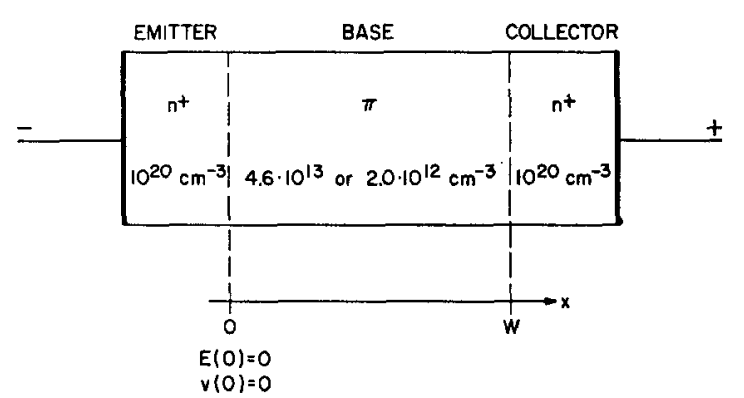

Fic. 1. Schematic representation of the $n^{+} \pi n^{+}$structure and the doping concentrations used in the three regions of the device. The coordinate system and the boundary conditions assumed in the analysis are shown as well.

has been selected for this work in the form of an $n^{+} \pi n^{+}$ device.

The theoretical models for which sclc has been analyzed in detail are normally one dimensional. They also always assume mathematically sharp boundaries. To provide a good basis of comparison, the device should therefore satisfy the requirements of planar geometry and abrupt junctions. To meet these conditions an appropriate single crystal of high perfection and low residual doping is crystallographically oriented into the $\langle 111\rangle$ direction and cut into slices which are then lapped and polished mechanically to a thickness of 20 to $60 \mu$. A final chemical etch with a mixture of 5 ppv conc. $\mathrm{HNO}_{3}$ and $1 \mathrm{ppv}$ conc. HF reduces this thickness further by approximately $10 \mu$. Abrupt junctions are obtained by diffusion of phosphorus into both surfaces of the slices. An open-tube arrangement is used at a diffusion temperature of $1150^{\circ} \mathrm{C}$ for $40 \mathrm{~min}$ and $\mathrm{P}_{2} \mathrm{O}_{5}$ as an impurity source. The depth of the junctions thus obtained is about $3 \mu$ with a surface concentration of the order of $10^{20} \mathrm{~cm}^{-3}$. Junction capacitance measurements as a function of reverse bias yield a voltage dependence of $C \approx \mathrm{V}^{-0.48}$ which indicates that the junction is indeed abrupt. The oxide layer created during the diffusion is removed with conc. HF, and a gold film of 0.1 to $0.3 \mu$ thickness is then evaporated on both sides of the slices at a pressure of approximately $10^{-6} \mathrm{Torr}$, and a temperature of $250^{\circ} \mathrm{C}$. The temperature is then raised to $350^{\circ} \mathrm{C}$ for $5 \mathrm{~min}$. The resulting electrical bond is stable, linear, and of a residual series resistance small enough to be negligible in all measurements. In a final step, the slices are divided into wafers of an area of $1-2 \times 10^{-3} \mathrm{~cm}^{2}$. This small size is chosen in an effort to achieve high current densities while maintaining moderate current levels at the signal source. The best results are obtained by simply breaking the slices, but masking and etch-cutting also works. A cross section of a wafer is represented schematically in Fig. 1. Details on the sample prepararation are given elsewhere. ${ }^{14}$

The comparison of the experimental results with the theory requires the knowledge of the physical pa-

${ }^{14} \mathrm{~S}$. Denda and M-A. Nicolet, JPL Space Programs Summary No. 37-33, Vol. IV (1965), p. 57. rameters of each wafer. The base thickness $W$ is obtained from metallurgical cuts by standard procedures of potting, wedge-lapping, and polishing after all measurements are completed. Photographs are taken through a metallurgical microscope and measured. The final accuracy is $\pm 15 \%$. The same photographic technique is used to determine the area of a sample. The error here is less than $5 \%$. The residual impurity concentration $N$ of the wafer may be changed by the heating cycle of the diffusion process. It is therefore necessary to measure $N$ on the completed samples. Three different methods were applied; (1) two point resistance measurements with a high input $(50 \mathrm{M} \Omega$ ) voltmeter on a reference bar passed through a diffusion cycle and subsequently stripped of its $n$ layers (only for $200 \Omega \cdot \mathrm{cm}$ crystal); (2) punch through voltage measurements, on samples of different base widths (only for $200 \Omega \cdot \mathrm{cm}$ crystal); (3) junction capacitance measurements versus reverse bias on the $n^{+} \pi$ junctions. The different results agree within $\pm 10 \%$. Two crystals of different origin were selected as base material. By manufacturer's specification their resistivities were $200 \Omega \cdot \mathrm{cm}$ and $2 \mathrm{k} \Omega \cdot \mathrm{cm}$. The residual doping $N$ determined on the samples after the diffusion was found to be $4.6 \times 10^{13} \mathrm{~cm}^{-3} \pm 10 \%$ and $2.0 \times 10^{12}$ $\mathrm{cm}^{-3} \pm 20 \%$ corresponding to resistivities of $300 \Omega \cdot \mathrm{cm}$ and approximately $8 \mathrm{k} \Omega \cdot \mathrm{cm}$, respectively.

\section{PRINCIPLE OF OPERATION}

An idealized $V-I$ characteristic of the device is sketched in Fig. 2. In general, three distinct ranges of operation exist. At low biases, the situation corresponds to that of a junction transistor with floating base (jtfb). In this jtfb range, the equilibrium condition in the population of minority and majority charge carriers in

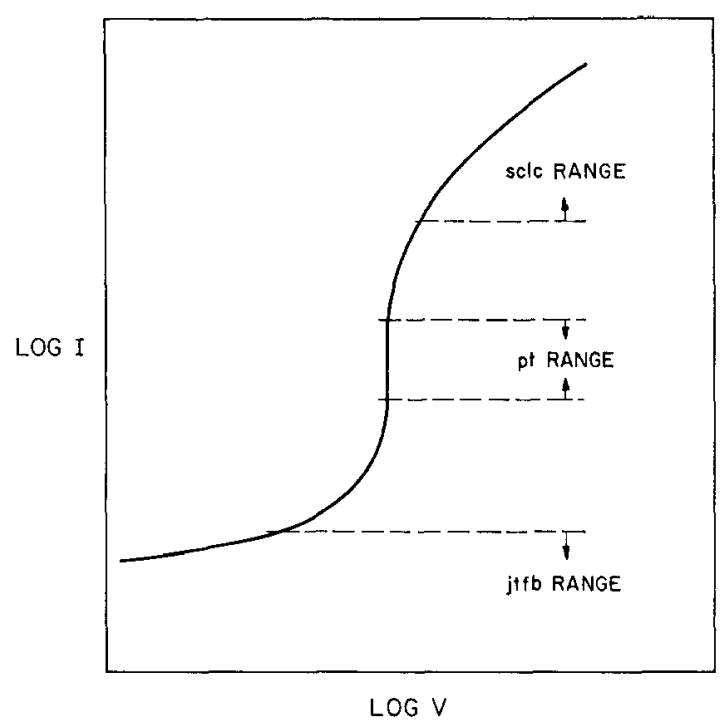

FIG. 2. Idealized $V-I$ characteristic of an $n^{+} \pi n^{+}$structure showing the three ranges of operation (junction transistor floating base, punch-through, and space-charge-limited current range). 


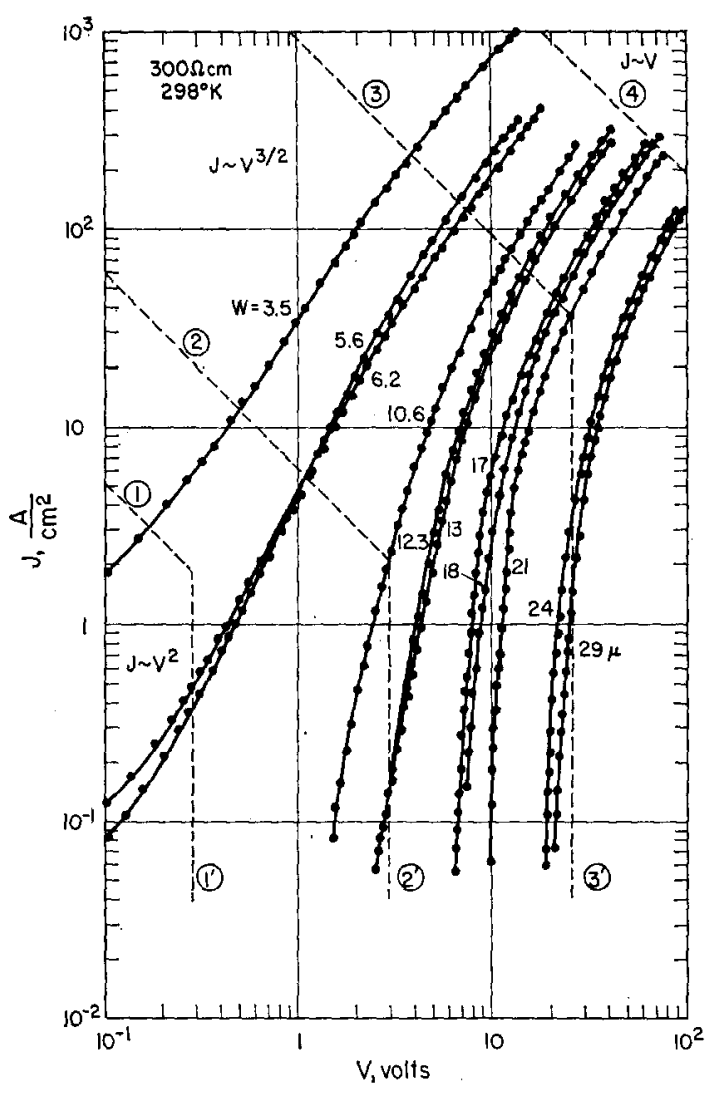

FIG. 3. V-I characteristics at room temperature of samples with $300-\Omega \cdot \mathrm{cm}$ base material.

the base is the determining factor. As the applied voltage increases, the widening depletion region of the reverse biased collector junction reduces the effective base width of the transistor. This increases the current amplification factor $b$ of the transistor and, therefore, the resulting net current through the device. Eventually, the collector depletion region reaches the emitter junction. At this "punch-through" voltage $V_{\mathrm{pt}}$ a base region as visualized in the junction transistor exists no more. From this point on, the emitter injects majority charge carriers (electrons) directly into the depletion region of the collector. As long as the net charge of these injected mobile electrons remains sufficiently small compared with the space charge of the fixed acceptors in the depletion region, the current increases rapidly without a significant increase in the applied voltage. This is the punch-through (pt) range of Fig. 2. As the concentration of injected mobile electrons increases, however, a point will be reached at which their space charge will dominate over that of the fixed acceptors. This is the range in which pure sclc flows. The over-all characteristic exhibits a striking similarity with that of scic in the presence of a trap filling voltage limit

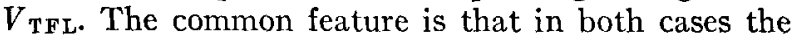
flow of pure sclc is initially inhibited by the presence of fixed space charge in the bulk. The origin of this space charge is, however, different in the two cases. In actual measurements of the $V-I$ characteristic short pulses must be used to avoid a temperature increase of the sample due to the power dissipated in it. An approximately linearly increasing voltage ramp with fast turn-off has been used at a repetition rate of 5-10 per second. The ramp was normally $20 \mu \mathrm{sec}$ long, a duration found experimentally to be short enough to eliminate any observable heating of the sample and long enough to avoid transient phenomena in sample or circuit. The sweep generator of an oscilloscope served as primary signal source. After passing through two cathode followers, the signal had a maximum amplitude of $120 \mathrm{~V}$ at $0.2 \mathrm{~A}$.

The voltage across the sample and the current flowing through it are displayed on the $x$ and $y$ axes of an oscilloscope and recorded photographically or visually point by point. The sample itself is placed between the goldplated base of a holder and a gold-plated finger attached to a micromanipulator. Electrical connection is established by applying slight mechanical pressure. Measurements below room temperature are performed by simply immersing the holder in liquid nitrogen or in isopentane cooled to the desired temperature by adding some liquid nitrogen to it.

Samples whose characteristics were not symmetrical with respect to an inversion of the applied bias were rejected.

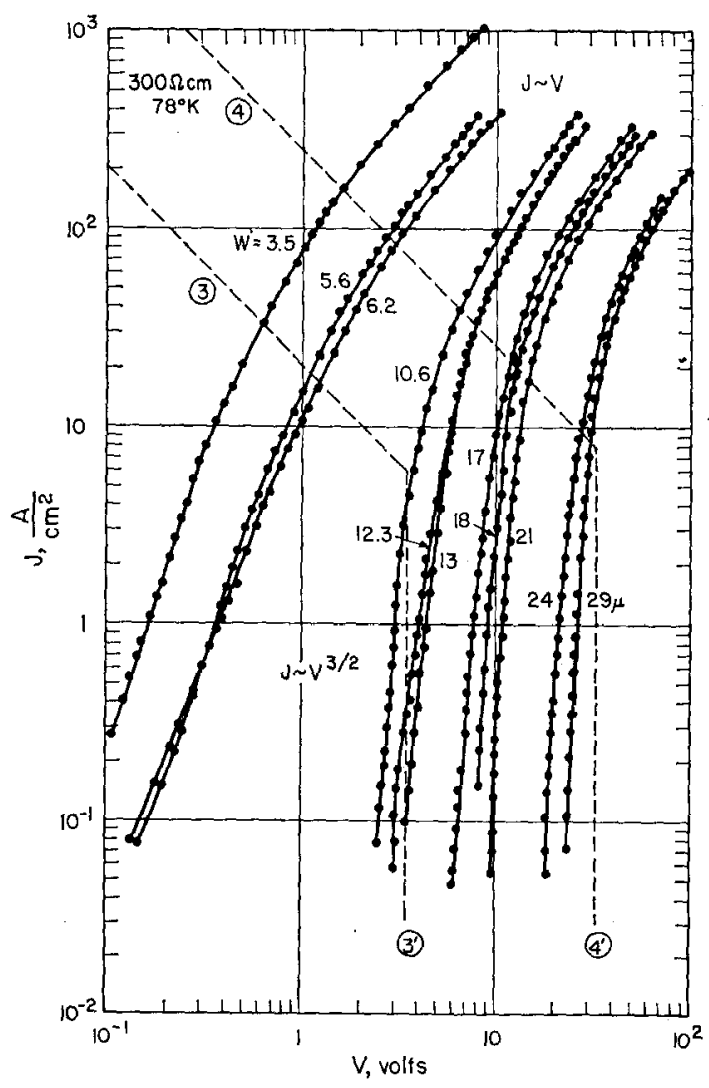

FIG. 4. V-I characteristics of samples at $78^{\circ} \mathrm{K}$ with $300-\Omega \cdot \mathrm{cm}$ base material. 


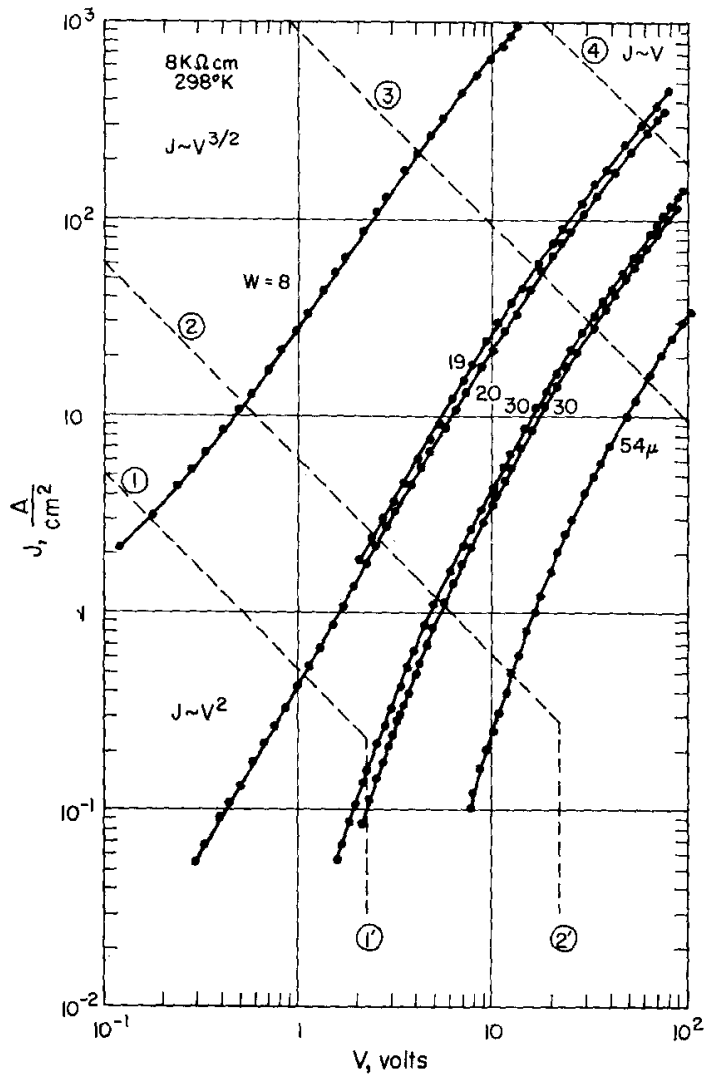

FIG. 5. V-I characteristics of samples at room temperature with $8-\mathrm{k} \Omega \cdot \mathrm{cm}$ base material.

\section{V-I CHARACTERISTICS AND COMPARISON WITH THEORY}

\subsection{Experimental Results}

Figure 3 shows a family of $V-I$ characteristics obtained at room temperature on the $300-\Omega \cdot \mathrm{cm}$ base material with the base width as parameter. The characteristics of the same samples at liquid-nitrogen temperature are given in Fig. 4. Corresponding results obtained on the $8-\mathrm{k} \Omega \cdot \mathrm{cm}$ base material are shown in Figs. 5 and 6 . The dashed lines in these figures refer to the temperature of the free charge carriers, as discussed in Sec. 4.4.

As can be seen, thicker samples clearly exhibit a punch-through range. In thinner samples the transition from the jtfb range to the sclc range is continuous, with a tendency for the pt range to be more distinct at lower temperatures. In a less pronounced fashion, this phenomenon has been observed before in germanium by Shumka. ${ }^{6}$ It is believed to be related to the finite width of the transition region of the $p n$ junctions at thermal equilibrium and the existence of a "virtual cathode." But minority charge carriers may also contribute to this effect, as indicated by the influence of white light incident on the sample. The $V-I$ characteristics of structures with a clean punch-through remained unaffected by light, whereas the others were noticeably modified in their lower current ranges.
A detailed analytical treatment of the transition from the jtfb range to the pt range does not exist in the literature. An analysis of the case in which this transition leads simultaneously into the sclc range does not exist either and is likely to be even more complex than the former. For this reason attention is focused primarily on the sclc and the pt ranges of the $V-I$ characteristics, that is, on the upper and the right-hand halves of Figs. 3-6, with only occasional reference to the lower left corner of these plots.

\subsection{Field-Dependent Mobility}

All results published so far on pure sclc in high-mobility materials such as germanium and silicon show clearly that the departure of the velocity distribution of the free charge carriers from that of thermal equilibrium with the lattice is significant for a proper interpretation of the characteristics of sclc. A good number of authors have studied the changes that occur in the velocity distribution of charge carriers at high electric field strengths, but only Ryder, ${ }^{15}$ Prior, ${ }^{16}$ and Jorgensen et al. ${ }^{17}$ give experimental results for electrons in silicon. Figure 7 reproduces measurements of Ryder and

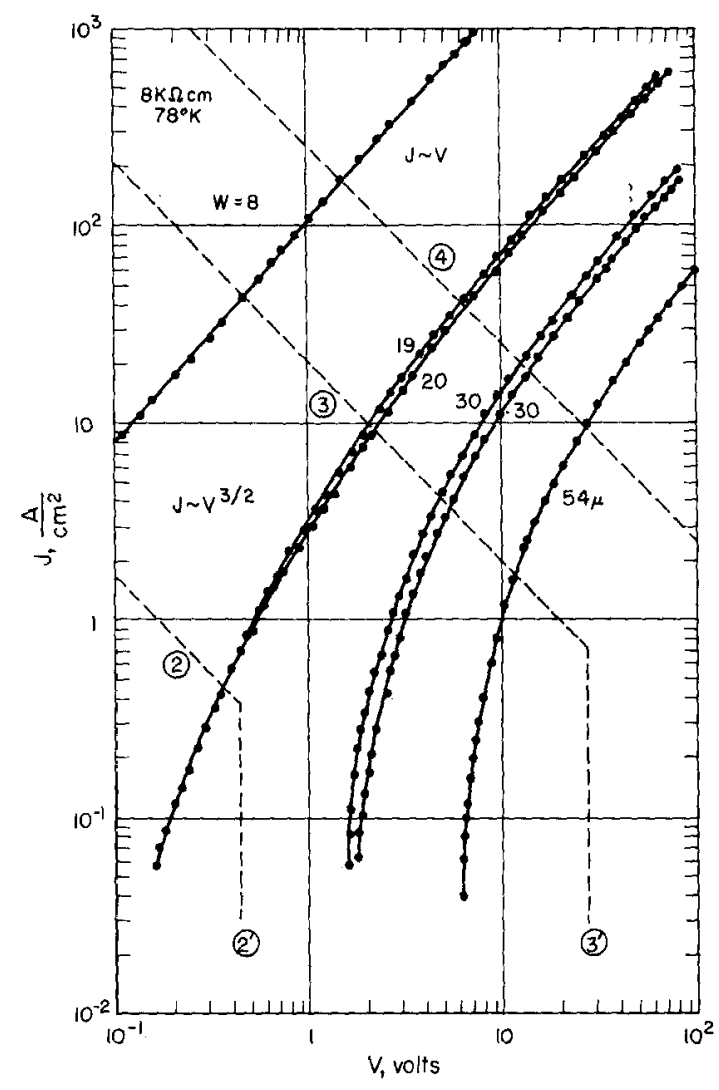

Fig. 6. $V-I$ characteristics of samples at $78^{\circ} \mathrm{K}$ with $8-\mathrm{k} \Omega \cdot \mathrm{cm}$ base material.

${ }^{15}$ E. J. Ryder, Phys. Rev. 90, 766 (1953).

${ }^{16}$ A. C. Prior, J. Phys. Chem. Solids 12, 175 (1959).

${ }^{17} \mathrm{M}$. H. Jørgensen, N. I. Meyer, and K. J. Schmidt-Tiedemann, Solid-State Commun. 1, 226 (1963). 


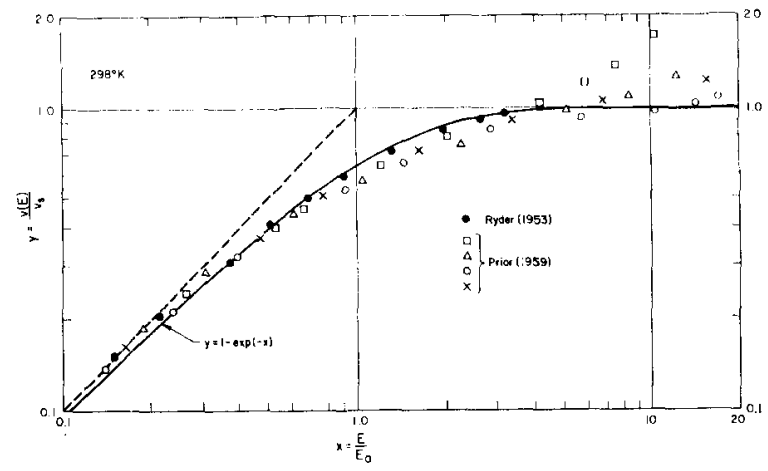

FIG. 7. Experimental results of Ryder ${ }^{15}$ and Prior ${ }^{16}$ on the field dependence of the drift velocity of electrons in silicon at room temperature reproduced in a normalized plot with $E_{0}=6.5 \times 10^{3}$ $\mathrm{V} / \mathrm{cm}$ and $\mu_{0} E_{0}=v_{s}=9.0 \times 10^{6} \mathrm{~cm} / \mathrm{sec}$. To a first approximation a complementary inverse exponential describes the results quite well over most of the field range.

Prior on the field dependence of the drift velocity. One notices that the electron mobility decreases progressively from its low field value. But no clear range of constant slope exists in which $\mu \approx E^{-\frac{1}{2}}$. There also is no conclusive evidence establishing the existence of a range of complete velocity saturation. Simple theories predict, however, that these ranges exist and the experimental velocity-field relationship of Fig. 7 has therefore been interpreted at times in terms of a hypothetical dependence as shown in Fig. $8^{., 15}$ The advantage of this simplified form is its straightforward analytical description. Most of the discussion to follow is based on this model of the velocity-field dependence, using the definitions of the low field mobility $\mu_{0}$, the critical electric field $E_{c}$, the critical drift velocity $v_{c}$, the saturation field $E_{s}$, the saturation velocity $v_{s}$, and of $E_{0}$ as indicated in this figure.

An equally convenient approach to obtain a simple analytical description of the velocity-field relationship is to find an elementary function which adequately reflects the general trend of the experimental results of Fig. 7. Several elementary functions have been tested on this property. The complementary reciprocal exponential was found most appropriate. It is also represented in Fig. 7. The location with respect to the experimental points is arbitrary within a shift along the line of unit slope because of the doubly logarithmic scale. The curve has been drawn where the best over-all agreement is obtained. In that position $E_{0}=6.5 \times 10^{3}$ $\mathrm{V} / \mathrm{cm}$ and $v_{s}=9.0 \times 10^{6} \mathrm{~cm} / \mathrm{sec}$. As can be seen, this function provides a reasonably satisfactory representation except at the highest field strengths. But the same restriction also applies to the function of Fig. 8 . Furthermore, the experimental points at the highest field strengths are subject to some uncertainties and the misfit between assumed and true velocity-field dependence may not be much larger there than at lower field strengths.

There is a criticism of a more fundamental nature which may be advanced with regard to the use of the results of Fig. 7 in the analysis of pure sclc. These results have been obtained on homogeneous pieces of bulk crystals under the action of a homogeneous electric ficld at steady state. In an $n^{+} \pi n^{+}$structure operated in the sclc range, on the other hand, both the field distribution and the charge carrier concentration vary extremely rapidly over distances of the order of $10 \mu$, even under steady-state conditions. In addition, the model of the $n^{+} \pi n^{+}$structure used in the analysis includes other simplifying assumptions of its own. It is difficult to assess the relative significance of these various simplifications. The analytical representations of Figs. 7 and 8 may therefore well be sufficient or even good in the present context. The complementary reciprocal exponential of Fig. 7 is used in the discussion of the temperature dependence of pure sclc.

\subsection{Models of Pure sclc}

The simplest model against which the experimental results are compared is based on the following significant assumptions: (1) There are no imperfections and defects of any kind in the solid, and its intrinsic resistivity is infinite; (2) the emitter has an infinite abundance, that is, it can maintain an unlimited concentration of charge carriers at its boundary; (3) the charge carriers are injected into the base with vanishing initial velocity; (4) the injected charge carriers are transported through the base by drift only - diffusion is ignored; (5) all variables are functions of one coordinate only. Figure 1 shows the convention adopted for the coordinate and the description of the geometrical parameters of the model. Under these conditions, the flow of a current through the base is described by the following equations and conditions at steady state:

$$
\begin{aligned}
d E(x) / d x & =\rho(x) / \epsilon \epsilon_{0}, \\
d \phi(x) / d x & =-E(x), \\
J & =\rho(x) \cdot v(x), \\
E(0) & =0, \\
\phi(0)-\phi(W) & =V,
\end{aligned}
$$

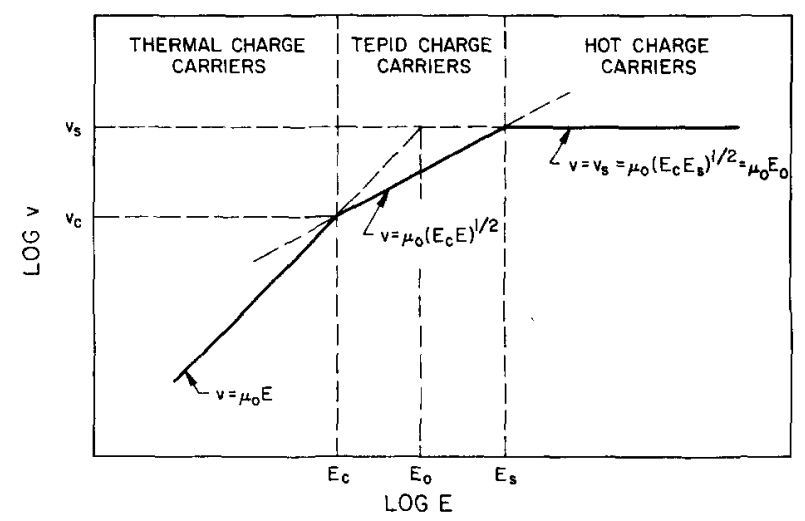

Fig. 8. The dependence of the drift velocity on electric field strength assumed to represent the experimental results of Fig. 7 in simple analytical terms. 


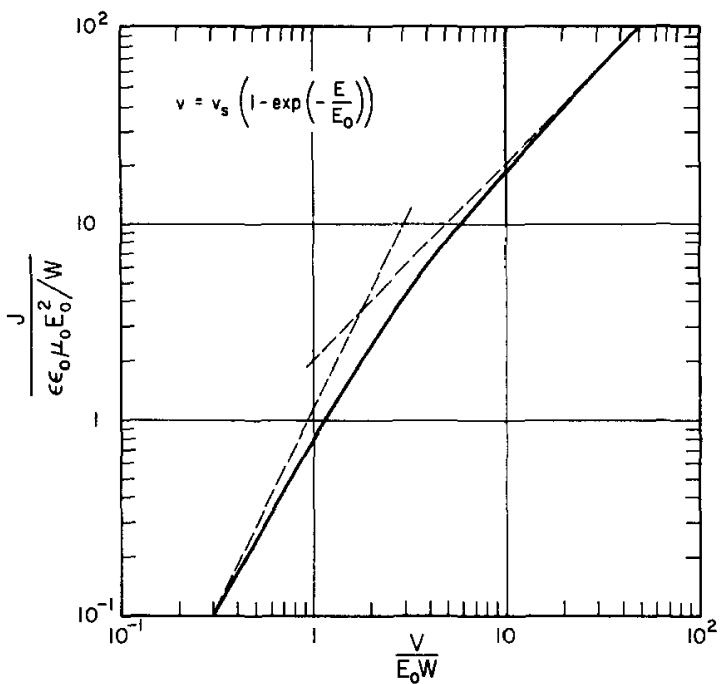

FIG. 9. Theoretical $V-I$ characteristic of pure sclc derived from the model of Sec. $4.3^{\circ}$ and a field dependence of the drift velocity as given in Fig. 7 and Eq. (7).

where the symbols have their conventional meaning and positive charge carriers are assumed for convenience. The velocity-field relationship takes the forms

$$
\begin{aligned}
& v(x)=\mu_{0} E(x), \quad E<E_{c} ; \\
& v(x)=\mu_{0}\left[E_{c} E(x)\right]^{\frac{1}{2}}, \quad E_{c}<E<E_{s} ; \\
& v(x)=\mu_{0}\left(E_{c} E_{s}\right)^{\frac{1}{2}}=v_{s}, \quad E_{s}<E ;
\end{aligned}
$$

or

$$
v(x)=v_{s}\left[1-\exp \left(-E / E_{0}\right)\right]
$$

depending on whether the description of Fig. 8 or 7 is chosen. With Eq. (6) an exact solution can be obtained by subdividing the base into regions corresponding to Eqs. (6a), (6b), and (6c) and subsequently matching the different solutions at their respective

TABLE I. Functional dependence of the electric field $E(x)$ and of the absolute value of the electrostatic potential $\phi(x)$ valid in the asymptotic range of the $V-I$ characteristics of Fig. 10. The models correspond to that of Mott and Gurney ${ }^{1}$ and neglect any fixed space charge in the base but assume either thermal, tepid, or hot charge carriers. The value of the field strength at any point

\begin{tabular}{|c|c|c|c|}
\hline $\begin{array}{c}\text { Charge } \\
\text { carrier } \\
\text { distribution }\end{array}$ & Thermal & Tepid & Hot \\
\hline$E(x)$ & $\left(2 \frac{J}{\epsilon \epsilon_{0} \mu_{0}}\right)_{(8)}^{3}$ & $\left(\frac{3}{2} \cdot \frac{J}{\epsilon \epsilon_{0} \mu_{0} E_{c}}\right)_{(9)}^{\prime} x^{\frac{3}{3}}$ & $\frac{J}{-x}-x$ \\
\hline$|\phi(x)|$ & $\frac{2}{3}\left(2 \frac{J}{\epsilon \epsilon_{0} \mu_{0}}\right)^{t}$ & $\frac{3}{5}\left(\frac{3}{2} \cdot \frac{J}{\epsilon \epsilon_{0} \mu_{0} E_{c}^{\frac{1}{2}}}\right)^{3} x^{5 / 3}$ & $\frac{1}{2} \cdot \frac{J}{\epsilon \epsilon_{0} y_{s}} x^{2}$ \\
\hline
\end{tabular}
of a sample of Figs. 3-6 is obtained readily from the first line of this table by noting that $E(\mathrm{~W})=c(\mathrm{~V} / \mathrm{W})$, where the constant $c$ is $\frac{3}{2}$ for thermal, $5 / 3$ for tepid and 2 for hot charge carriers. boundaries. The approach is tedious and the final over-all result unwieldy. If Eq. (7) is used, the subdivision of the base in different regions is avoided, but numerical methods must be resorted to for explicit solutions. The $V-I$ characteristic of pure sclc obtained by such a numerical integration is shown in Fig. 9. Details of these calculations are omitted here.

The mathematical difficulties disappear if Eq. (6a) is assumed to be valid throughout, that is, if deviations from Ohm's law are neglected. The resulting functional dependences of the electric field $E(x)$ and the potential $\phi(x)$ are reported in Table I. This is the model originally proposed by Mott and Gurney. ${ }^{1}$ The form of Eq. (6) immediately suggests two modifications of this model by substituting Eq. (6b) or (6c) for (6a). Table I includes these two additional cases as well. It is seen that the three models yield $V-I$ characteristics for pure sclc which are power laws of $V^{2}, V^{\frac{3}{2}}$, and $V$ for thermal, tepid, ${ }^{18}$ and hot charge carriers, respectively: This agrees with a general rule recently enunciated by Lee. ${ }^{19}$

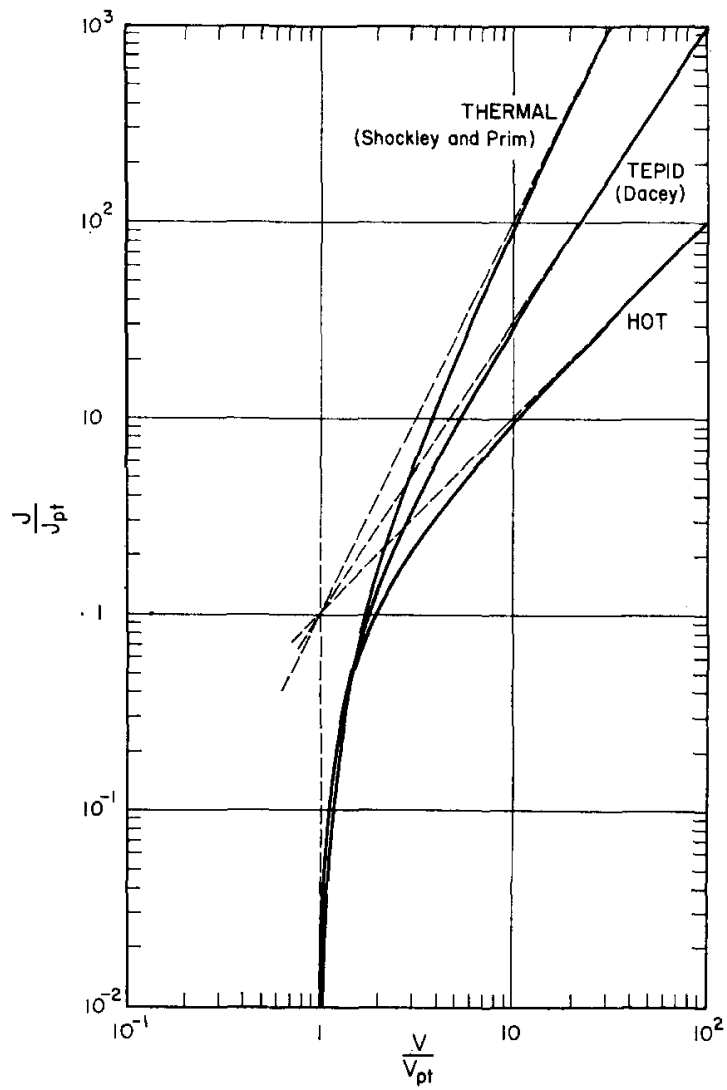

FIG. 10. Theoretical $V-I$ characteristics of pure sclc derived from the model of Sec. 4.3 and assuming thermal [Eq. (6a)], tepid $[\mathrm{Eq} .(6 \mathrm{~b})]$ or hot $[\mathrm{Eq} .(6 \mathrm{c})]$ charge carriers, corresponding to the three regions of Fig. 8.

${ }^{18}$ The term is used here to refer to charge carriers departing significantly from conditions of both thermal equilibrium and drift velocity saturation (see Fig. 8).

${ }_{19}^{19}$ D. H. Lee and M-A. Nicolet, Solid-State Electron. 8, 182 (1965). 
The models proposed so far are, however, still inadequate because they ignore the actual conditions prevailing in the base at thermal equilibrium. If these conditions are included, the mathematical obstacles increase to the extent that a less accurate, but more flexible approach seems more promising.

Pursuing this thought, Shockley and Prim $^{20}$ have proposed a model in which the fixed space charge of the residual doping concentration $N$ in the base is retained, but in which Eq. (6a) is assumed to be valid throughout, as is done in the model of Mott and Gurney. The result is a $V-I$ characteristic in which both a pt range and a sclc range appear (see Fig. 10). Dacey ${ }^{5}$ has introduced the effect of field-dependent mobility in this same model by substituting (6b) for (6a). An obvious third possibility is to assume velocity saturation throughout the base and use (6c). The three $V-I$ characteristics derived from these three variants are represented in Fig. 10. Voltages are normalized with respect to $V_{\mathrm{pt}}=q N W^{2} / 2 \epsilon \epsilon_{0}$, the punch-through voltage as obtained from the simple full depletion approximation of the reverse-biased collector junction. Currents are normalized with respect to that particular pure sclc density $J_{\mathrm{pt}}$ that would flow in each particular model if the applied voltage were equal to $V_{\mathrm{pt}}$ and if the base were free of fixed space charge; e.g.

$$
\begin{aligned}
& J_{\mathrm{pt}}=(9 / 8) \epsilon_{0} \mu_{0} V_{\mathrm{pt}}{ }^{2} / W^{3} \quad \text { for } \\
& \text { thermal charge carriers, (6a); } \\
& J_{\mathrm{pt}}=\frac{2}{3}(5 / 3)^{\frac{3}{2}} \epsilon_{0} \mu_{0} E_{\mathrm{c}}{ }^{\frac{1}{3}} V_{\mathrm{pt}} \mathrm{t}^{\frac{3}{2}} / W^{\frac{5}{2}} \text { for } \\
& \text { tepid charge carriers, (6b); } \\
& J_{\mathrm{pt}}=2 \epsilon \epsilon_{0} v_{s} V_{\mathrm{pt}} / W^{2}=v_{s} q N \quad \text { for } \\
& \text { hot charge carriers, (6c). }
\end{aligned}
$$

It is seen that for the three cases of thermal, tepid, and hot charge carriers the asymptotic dependences of the $V-I$ characteristics are power laws of $V^{2}, V^{\frac{3}{2}}$, and $V$, respectively. They are the characteristics of the simple models discussed above in which fixed space charge is absent in the base.

\subsection{Matching Characteristics of Models and Device}

The theoretical characteristics shown in Fig. 10 are now matched to the experimental results. The closeness of the fit will be a measure of the accuracy of the models and they, in turn, yield information on the nature of the current flow in the device.

It is obvious that in the presence of a velocity-field relationship as shown in Fig. 7 the theoretical characteristics of thermal and tepid charge carriers in Fig. 10 can not apply in their asymptotic limits. In the transition region between the pt range and the sclc range, on the other hand, the general trend of the three curves of Fig. 10 is very much the same. To properly match experimental and theoretical curves, a procedure

\footnotetext{
${ }^{20}$ W. Shockley and R. C. Prim, Phys. Rev. 90, 753 (1953).
}

\begin{tabular}{|c|c|c|c|c|}
\hline Boundary & Relationship & & & \\
\hline 1 & $V J=\frac{1}{3} \epsilon \epsilon_{0} \mu_{0} E_{c^{3}}$ & & & \\
\hline $1^{\prime}$ & $V=\varepsilon_{\epsilon_{0}} E_{c_{c}^{2}} / 2 q N$ & & & \\
\hline 2 & $V J=4 \epsilon \epsilon_{0} \mu_{0} E_{c} c^{a}$ & & at $298^{\circ} \mathrm{K}$ & at $78^{\circ} \mathrm{K}$ \\
\hline $2^{\prime}$ & $V=5 \epsilon \epsilon_{0} E_{c}^{2} / q N$ & $\mu_{0}\left(\mathrm{~cm}^{2} / V_{s e c}\right)$ & 1400 & 10000 \\
\hline 3 & $V J=\frac{7}{\zeta} \in \epsilon_{0} \mu_{0} E_{c} c^{1 / 2} E_{d^{5}}{ }^{5 / 2}$ & $E_{e}(\mathrm{~V} / \mathrm{cm})$ & 1000 & 140 \\
\hline $3^{\prime}$ & $V=e \epsilon_{0} E_{\mathrm{a}}{ }^{2} / 2 q N$ & $E_{0}(\mathrm{~V} / \mathrm{cm})$ & 20000 & 7000 \\
\hline 4 & $V J=5 \epsilon \epsilon_{0} \mu E_{\mathfrak{c}}^{1 / 2} E_{s^{5}}^{5 / 2}$ & & & \\
\hline $4^{\prime}$ & $V=5 \epsilon_{0} E_{\mathrm{q}^{2}} / \mathrm{q} N$ & & & \\
\hline
\end{tabular}

TABLE II. Analytical expressions for the boundaries 1 and $1^{\prime}$ to 4 and $4^{\prime}$ in Figs. 3-6 and the numerical values assumed for the physical quantities involved. In the models used to derive these expressions, the electric field at the collector is constant along each of the boundaries.

is therefore desirable which will provide guidelines for a correct assignment. The dashed lines in Figs. 3-6 serve this purpose. They subdivide the figures into adjacent fields of operating points and are based on Fig. 8. If the maximum of the electric field strength in the base does not exceed the value of the critical electric field $E_{c}$, the theoretical $V-I$ characteristics of Fig. 10 based on a tepid and hot velocity distribution of the charge carriers cannot be valid. If, in addition, the current flowing through the device is small, the mobile charge carriers are negligible compared with the doping ions and the field maximum at the collector boundary can be derived from the assumption of a fully depleted base. This gives a limiting bias of $V=\epsilon_{0} E_{c}^{2} / 2 q N$. If, however, the current flowing through the device is large and the fixed space charge of the ionized doping atoms is negligible, the maximum of the electric field is given by Eq. (8) in Table I. Requiring that this value be at most equal to the critical field $E_{\mathrm{c}}$ yields a boundary of the form $V J \leq \frac{1}{3} \epsilon \epsilon_{0} \mu_{0} E_{c}^{3}$. The two boundaries $\mathrm{ob}-$ tained in this manner are those labeled $1^{\prime}$ and 1 in Figs. 3 and 5. They define a field in the lower left corner of the $V-I$ plane within which the theoretical curve for thermal charge carriers in Fig. 10 should apply and beyond which the same curve gradually loses its significance. A similar pair of boundaries 3 and $3^{\prime}$ can be found by analogous reasoning with $E_{s}$. They delineate a field of operating points within which the theoretical curve for tepid charge carriers should be used. In this case, however, there must obviously exist a limit towards lower operating points as well. This is the pair of boundaries 2 and $2^{\prime}$. They are derived from the condition that at the particular point between base and collector at which the field strength reaches the value of the critical field $E_{c}$ the voltage drop with respect to the emitter be at most $10 \%$ of the total applied voltage. The figure of $10 \%$ is arbitrary, but is felt to be of a correct order of magnitude in relation to the approximate significance of these boundaries. The lines marked 4 and $4^{\prime}$ have analogous meaning and delineate the field of operating points beyond which the theoretical curve of hot charge carriers should apply. The analytical representation of all these boundaries is given in Table II. The numerical values assumed for the physical quantities involved 


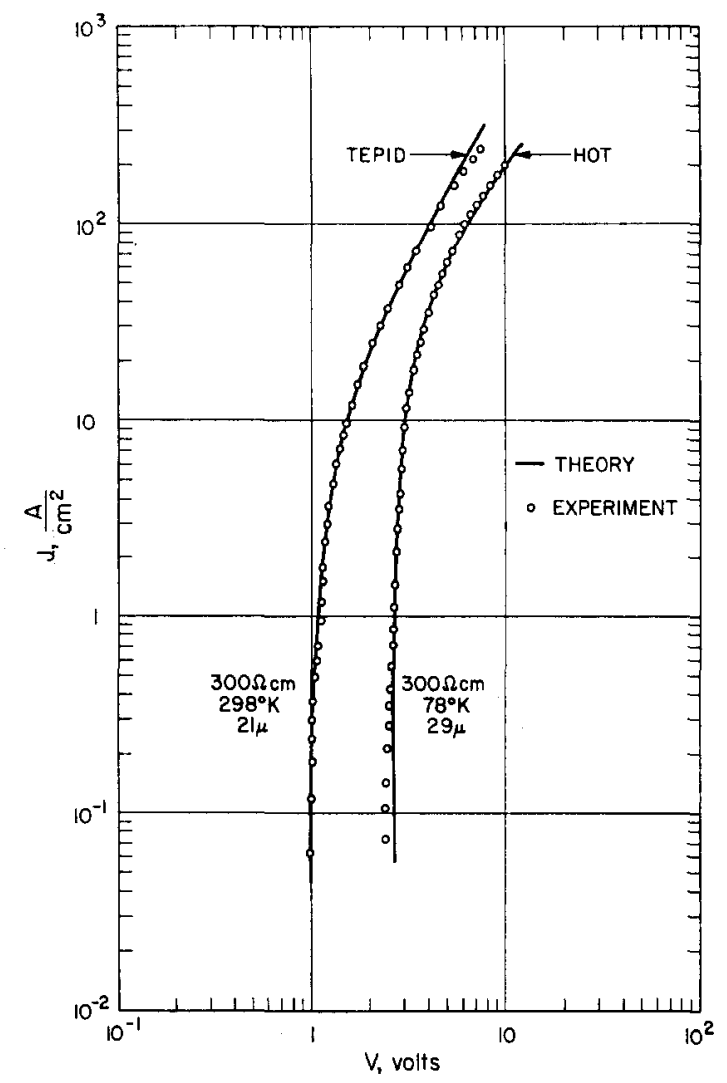

FIG. 11. Comparison of experimental results obtained on $300-\Omega \cdot \mathrm{cm}$ samples of 21 and $29-\mu$ base width at room temperature and at liquid-nitrogen temperature with the theoretical curves of Fig. 10 for sclc of tepid and hot charge carriers, respectively.

are reported in this same table. These figures are approximate only and were obtained from a preliminary analysis of the experimental results. In view of the approximate significance of the boundaries, further corrections were considered unnecessary.

As can be seen in Figs. 3-6 the experimental $V-I$ characteristics often cross several boundaries. In such a case no single theoretical curve of Fig. 10 is expected to give a complete fit. But if the experimental curve lies predominantly in one particular field, the corresponding theoretical curve should fit the data over most of its range. This is indeed the case for experimental curves with a clean punch-through. Two cases are shown in Fig. 11, where the experimental points and the proper theoretical curve at a position of best fit are represented in superposition.

The conclusion to be drawn from this agreement is that the models of Sec. 4.3 are good enough to adequately reproduce the main features of the dc characteristics of the $n^{+} \pi n^{+}$devices. More specifically, it is concluded that at $78^{\circ} \mathrm{K}$ and to some extent even at room temperature, the current flow at the highest operating points is carried by hot electrons in a state of complete velocity saturation. This statement is supported by further evidence presented in the next sections.

\subsection{Intersection Point of Asymptotes}

Each theoretical curve in Fig. 10 has an asymptote at high and at low current densities. The point of intersection $\left(J_{\mathrm{p} t}, V_{\mathrm{pt}}\right)$ of the two asymptotes is different for samples of different base widths. The equation for the locus of this point is obtained by eliminating the base width from the expression of the asymptotes. One finds for the locus of the theoretical curve of sclc:

with thermal charge carriers

$$
J_{\mathrm{pt}}=(9 / 8) \mu_{0}\left[(q N)^{3} / 8 \epsilon \epsilon_{0}\right]^{\frac{1}{2}} V_{\mathrm{pt}^{\frac{1}{2}}}=\text { const. } V_{\mathrm{pt}^{\frac{1}{2}}},
$$

with tepid charge carriers

$$
\begin{aligned}
J_{\mathrm{pt}}=\frac{2}{3}(5 / 3)^{\frac{3}{2}} \mu_{0}\left(\epsilon \epsilon_{0}\right)^{-\frac{1}{4}} E_{c^{\frac{1}{2}}}(q N / 2)^{5 / 4} V_{\mathrm{pt}}^{\frac{1}{4}} & \\
& =\text { const. } V_{\mathrm{pt}}^{\frac{1}{4}},
\end{aligned}
$$

and with hot charge carriers

$$
J_{\mathrm{pt}}=\mu_{0}\left(E_{c} E_{s}\right)^{\frac{1}{2}} q N=v_{s} q N=\text { const. }
$$

When the three loci are known experimentally, the three quantities $\mu_{0}, E_{c}$, and $E_{s}$ can be determined directly with the doping concentration $N$ as the only remaining parameter. The absence of a clear punchthrough range at low operating points (Fig. 5) unfurtunately precludes an experimental determination of $\mu_{0}$ from Eq. (15a) other than that of a mere estimation. The value found is, however, of the correct order of magnitude. A more favorable situation exists for a test of Eq. (15b). A number of experimental curves exhibiting a transition from the pt range to the sclc range with tepid charge carriers (between boundaries 2 and 3) are found in Fig. 3. But these curves also extend beyond the field of tepid charge carriers into that of hot charge carriers. This introduces uncertainties in the exact location of the theoretical curve of best fit and causes a fairly large error in the determination of the locus. The result is shown in Fig. 12. The $V^{\frac{1}{4}}$ line drawn into the figure shows that the experimental finding is at least consistent with Eq. (15b). From this

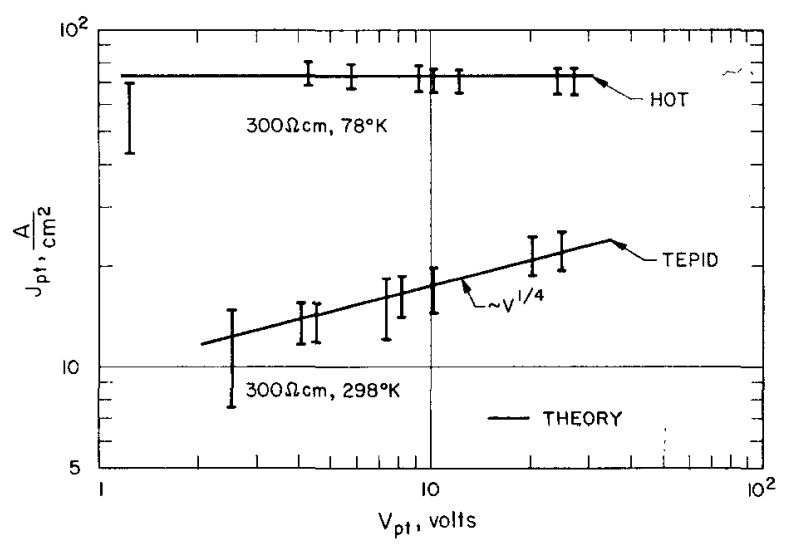

FIG. 12. The position of the intersection point $\left(V_{\mathrm{pt}}, J_{\mathrm{pt}}\right)$ of the two asymptotes of the theoretical characteristics of Fig. 10 for tepid and hot charge carriers when matched to the experimental curves of Figs. 3 and 4. 


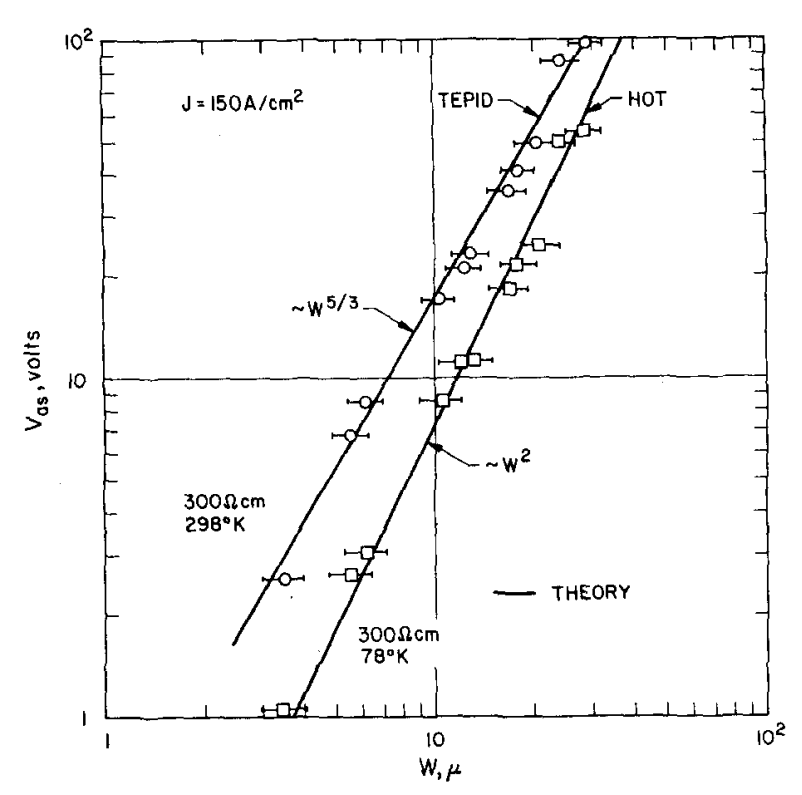

Fig. 13. The dependence upon the base width $W$ of the voltage $V_{\text {as }}$ of the asymptotes of pure sclc at a constant current density of $150 \mathrm{~A} / \mathrm{cm}^{2}$ obtained by matching the theoretical characteristics of Fig. 10 for tepid and hot charge carriers to experimental curves of Figs. 3 and 4 .

line, and with $\mu_{0}=1400 \mathrm{~cm}^{2} / V \mathrm{sec}$ a critical electric field of $E_{c}=930 \pm 250 \mathrm{~V} / \mathrm{cm}$ is derived. The large error is due to the strong dependence of $E_{c}$ on variations in the current density $J_{\mathrm{pt}}$. This value is significantly lower than would be anticipated from a simple straight-line approximation of Fig. 7 as sketched in Fig. 8. The difference may have to do both with the particular way in which a field-dependent drift velocity affects the $V-I$ characteristic of sclc and therefore with the method used to obtain this result as well as with the difficulty to define a critical electric field for a relationship as shown in Fig. 7. It is of interest to note in this connection that the theoretical $V-I$ characteristic of Fig. 9 is insensitive to minor changes in the field dependence of the carrier drift velocity. But these low values of $E_{c}$ raise questions regarding the usefulness and the final meaning of the concept of a critical electric field for electrons in silicon.

The uncertainties of matching the theoretical $V-I$ characteristic with the experimental ones are least for curves with a transition from the pt range to the sclc range with hot charge carriers (Fig. 4). The locus obtained from a best fit to these curves is also shown in Fig. 12 and agrees well with Eq. (15c). The straight line of constant current drawn through these points yields a saturation velocity $v_{s}$ for electrons in silicon at $78^{\circ} \mathrm{K}$ of $1.0 \times 10^{7} \mathrm{~cm} / \mathrm{sec}$ with an estimated error of approximately $10 \%$. But the $V-I$ characteristics themselves do not extend sufficiently far into the range of pure sclc to establish whether their slope is exactly unity or slightly larger. Further measurements are desirable in this range.
The saturation velocity of $1.0 \times 10^{7} \mathrm{~cm} / \mathrm{sec}$ at $78^{\circ} \mathrm{K}$ lies close to that of $9.0 \times 10^{6} \mathrm{~cm} / \mathrm{sec}$ extrapolated from the room-temperature measurements of Ryder and of Prior (Fig. 7). A similar finding is reported for germanium..$^{5}$ It is consistent with an interpretation of velocity saturation by optical phonon scattering.

\subsection{Dependence on Base Width}

Equations (11)-(13) predict that for a constant current density the asymptotes to the $V-I$ characteristics of pure sclc yield

$$
\begin{aligned}
& V_{\mathrm{as}} \approx W^{\frac{3}{3}} \text { for thermal charge carriers, } \\
& V_{\mathrm{as}} \approx W^{5 / 3} \text { for tepid charge carriers, } \\
& V_{\mathrm{as}} \approx W^{2} \text { for hot charge carriers. }
\end{aligned}
$$

To test these relationships a set of $V-I$ characteristics of pure sclc has to be given in each of the corresponding fields of the $V-I$ plane. The portion of the sclc range in the curves of Figs. 3 and 4 offer such sets for a test of Eqs. (16b) and (16c), respectively. Figure 13 shows the voltage value of the asymptotes to these curves at $150 \mathrm{~A} / \mathrm{cm}^{2}$. The two straight lines are computed from Eqs. (16b) and (16c) with $v_{s}=1.0 \times 10^{7} \mathrm{~cm} / \mathrm{sec}$, $\mu_{0}=1400 \mathrm{~cm}^{2} / \mathrm{Vsec}$, and $E_{\mathrm{c}}=1000 \mathrm{~V} / \mathrm{cm}$. The fit is excellent but possibly somewhat for tuitous, particularly in view of the uncertainty of the exact significance of the critical electric field $E_{c}$. The values of both $E_{c}$ and $v_{s}$ agree well with those obtained in the previous section. The estimated errors are $\pm 30 \%$ for $E_{c}$ and $\pm 10 \%$ for $v_{s}$, the difference being due to the nonequal powers with which $E_{e}$ and $v_{s}$ enter into Eqs. (14b) and (14c).

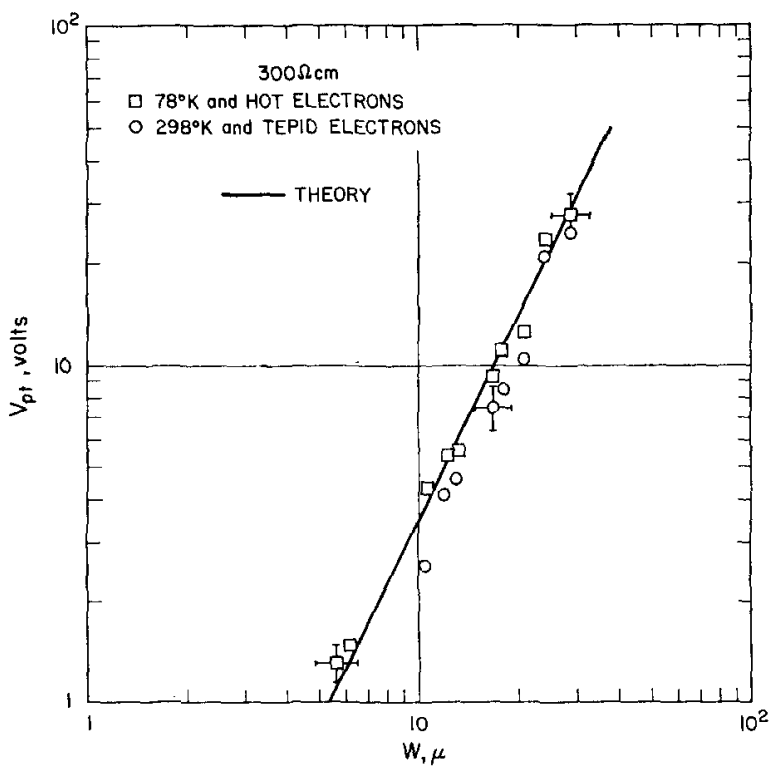

FIg. 14. The dependence upon base width $W$ of the punchthrough voltage $V_{\mathrm{pt}}$ given by the asymptote of the theoretical characteristics of Fig. 10 for tepid and hot charge carriers when matched to experimental curves of Figs. 3 and 4 . 
The correctness of the models used can also be tested in the pt range of the $V-I$ characteristics. According to the picture of full depletion of the base applicable in this range, the asymptote in the pt range of the theoretical curve of best fit must depend upon the base width as $V_{\mathrm{pt}}=\left(q N / 2 \epsilon \epsilon_{0}\right) W^{2}$. Figure 14 shows this dependence derived from the characteristics of Figs. 3 and 4 for the same set of curves used in Fig. 13. The straight line is the theoretical dependence computed with a value of $N=4.6 \times 10^{13} \mathrm{~cm}^{-3}$. The agreement is excellent for the characteristics of sclc with hot electrons. The results derived from the characteristics of sclc with tepid electrons deviate slightly in a systematic manner. This error can be accounted for easily by the uncertainty in the true position of the theoretical curve due to its systematic deviation from the experimental curve at the high current end.

\section{TEMPERATURE DEPENDENCE}

Pure sclc depends upon the temperature only through variations of the dielectric constant and the drift velocity of the charge carriers. In high-purity silicon, the former is negligible compared to the latter. The changes of sclc with temperature therefore reflect the temperature dependence of the velocity-field relationship.

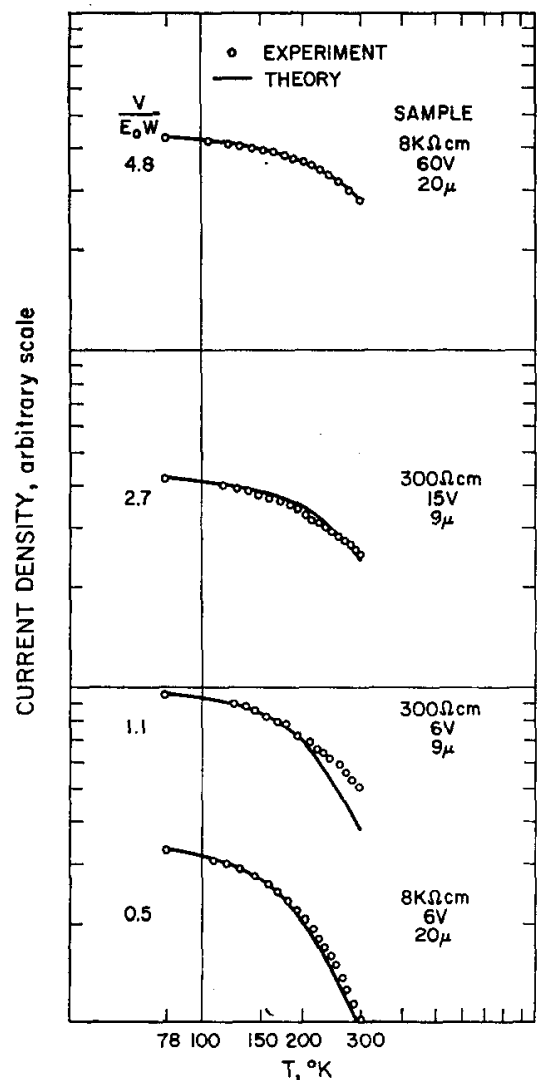

Fig. 15. Temperature dependence of current density observed at constant applied voltage for two different samples at two different operating points.
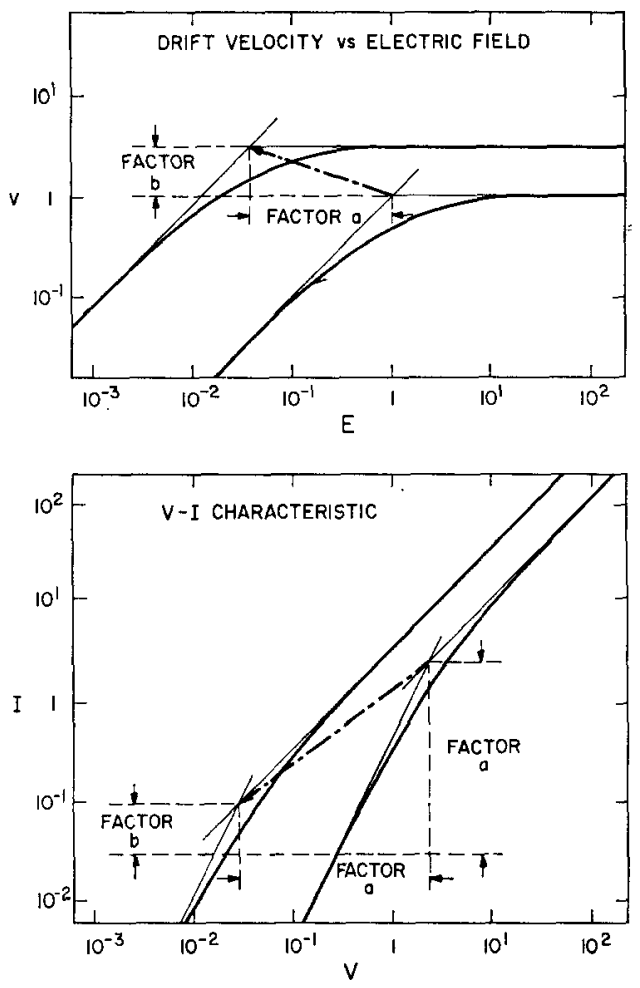

FIG. 16. When the curve of the velocity-field relationship is shifted in a doubly logarithmic plot (top) without change of form, the $V-I$ characteristic is displaced in a doubly logarithmic plot (bottom) without change of form either. The numerical relation between the two shifts is given by the two arrows.

They also provide means to further test the models proposed above.

Figure 15 shows the variation of the current with temperature at constant applied voltage for two samples. (The $V-I$ characteristics of these samples are not shown in Figs. 3-6). The operating points have been selected such as to fall into different fields of the $V-I$ plane at room temperature. It is seen that in the field of hot charge carriers the current increases only lightly with decreasing temperature, whereas for tepid charge carriers the variation is more pronounced. In no case, however, is there an increase of current in form of a power law of $T^{-2.5}$ indicative of thermal charge carrier mobility variation. ${ }^{21}$ This is not surprising since none of the operating points fall into the field of thermal charge carriers. One notices also that the operating points of a sample at $78^{\circ} \mathrm{K}$ and at room temperature fall into different fields of the $V-I$ plane. This indicates a change in the state of the velocity distribution of the electrons. To properly analyze the temperature variation observed, a model is therefore needed which includes continuous changes in the velocity distribution of the charge carriers. The $V-I$ characteristic of Fig. 9 satisfies this requirement because it is based on Eq. (7), Fig. 7, rather than on Eq. (6), Fig. 8. The disadvantage of

${ }^{21}$ G. W. Ludwig and R. L. Watters, Phys. Rev. 101, 1699 (1956). 
this solution is that punch-through effects are not included. For this reason most of the temperature measurements reported in Fig. 15 are restricted to operating points in the sclc range of the $V-I$ characteristics, where effects of punch-through are negligible.

The early experiments of Ryder ${ }^{15}$ and the present ones indicate that the saturation velocity $v_{8}$ of hot electrons is only slightly temperature dependent. On the other hand, the drift velocity of thermal electrons in pure silicon crystals varies approximately as a power law in the temperature range considered here. Both these facts can be included in an interpretation of Fig. 15 by noticing that a simple shift of the velocity-field curve in a doubly logarithmic plot will generate another shift of the $V-I$ curve if also plotted in a doubly logarithmic scale. Figure 16 gives a graphical sketch of this interconnection. The rule follows from the doubly logarithmic and normalized nature of the scales in the solution of Fig. 9. If it is now assumed that the temperature causes a shift of the velocity-field relationship as just described, the theoretically expected variation of the current at constant voltage then follows readily by graphical construction.

The main attribute of the method is its simplicity. Ryder's results indicate that in germanium this model is at least as good as the assumption of a complementary reciprocal exponential for the velocity-field relationship. A similar situation is likely to hold for silicon, but to the knowledge of the authors, no information on the driftvelocity dependence of electrons in the temperature range of $78^{\circ} \mathrm{K}$ to room temperature is available. There is little doubt, however, that the model includes the

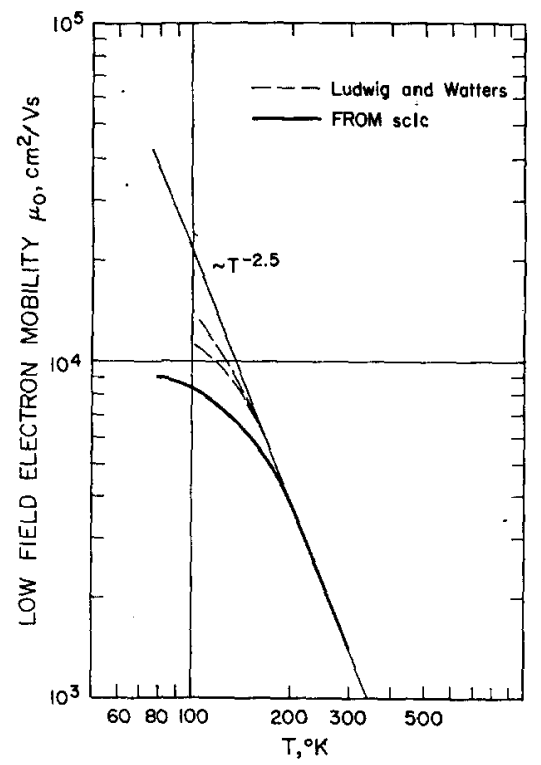

FIG, '17. Temperature dependence of the low field mobility of electrons deduced from the temperature dependence of pure sclc. Results of Ludwig and Watters ${ }^{21}$ obtained on $180 \Omega \cdot \mathrm{cm}$ (upper curve) and $45 \Omega \cdot \mathrm{cm}$ (lower curve) $n$-type crystals are included for comparison. main features of the true situation. Furthermore, an analysis shows that the $V-I$ characteristic of Fig. 9 is insensitive to small changes of the velocity-field relationship. As long as the effect of temperature on this relationship is predominantly one of scaling, the model must therefore give quantitative results which approximate the actual situation closely.

By successive approximations a best fit to the experimental results was obtained with a factor $b$ (see Fig. 16) of 1.25 between room temperature and $78^{\circ} \mathrm{K}$ and a temperature dependence of the factor $a$ corresponding to a low field mobility variation as shown in Fig. 17. The theoretical temperature dependence of pure sclc obtained in this fashion is compared with the measurements in Fig. 15. Deviations are noticeable only for the $300-\Omega \cdot \mathrm{cm}$ sample of $9 \mu$ with $6-\mathrm{V}$ bias at room temperature. Figure 3 shows, however, that this operating point no longer falls into the field of pure sclc but approaches the pt range of the $V-I$ characteristics, so that a disagreement is actually expected in this case. The factor $b$ and the temperature dependence of the lowfield mobility estabilshed by this method are not very accurate. An uncertainty of approximately $20 \%$ is believed to constitute a representative error. In view of this fact, the ratio of 1.25 compares fairly with the values of $1.0 \times 10^{7} \mathrm{~cm} / \mathrm{sec}$ and $9.0 \times 10^{6} \mathrm{~cm} / \mathrm{sec}$ found previously for the saturation velocity at $78^{\circ} \mathrm{K}$ and room temperature. The dependence of the low-field mobility with temperature also agrees rather well in the highertemperature range with the results of Ludwig and Watters ${ }^{21}$ reproduced in Fig. 17 for comparison. At low temperatures significant deviations exist both from the $T^{-2.5}$ dependence as well as from Ludwig and Watters' results. From arguments of crystal purity and impurity scattering, a difference from sample to sample is expected in this temperature range. But Ludwig and Watters' samples were of resistivities less than 200 $\Omega \cdot \mathrm{cm}$ while the present ones are above this limit and should therefore exhibit electron mobilities higher than those measured by these authors. In this respect, the simultaneous evaluation of the temperature dependence of $8-\mathrm{k} \Omega \cdot \mathrm{cm}$ and $300-\Omega \cdot \mathrm{cm}$ samples may be questioned. More eshaustive and systematic measurements than those of Fig. 15 will have to be performed to clarify this point. Until such a time the present treatment must be regarded as preliminary.

\section{DISCUSSION AND CONCLUSION}

The findings reported above offer a consistent picture. The theoretical models adopted are simple but adequate. With a possible exception for the temperature dependence, the analysis is quantitative and yields values for the saturation velocity $v_{s}$ of electrons at $78^{\circ} \mathrm{K}$ and for the critical electric field $E_{c}$, as far as such a notion is meaningful for electrons in silicon.

In view of this apparent success it is of interest to note that with a minor exception in the treatment of the 


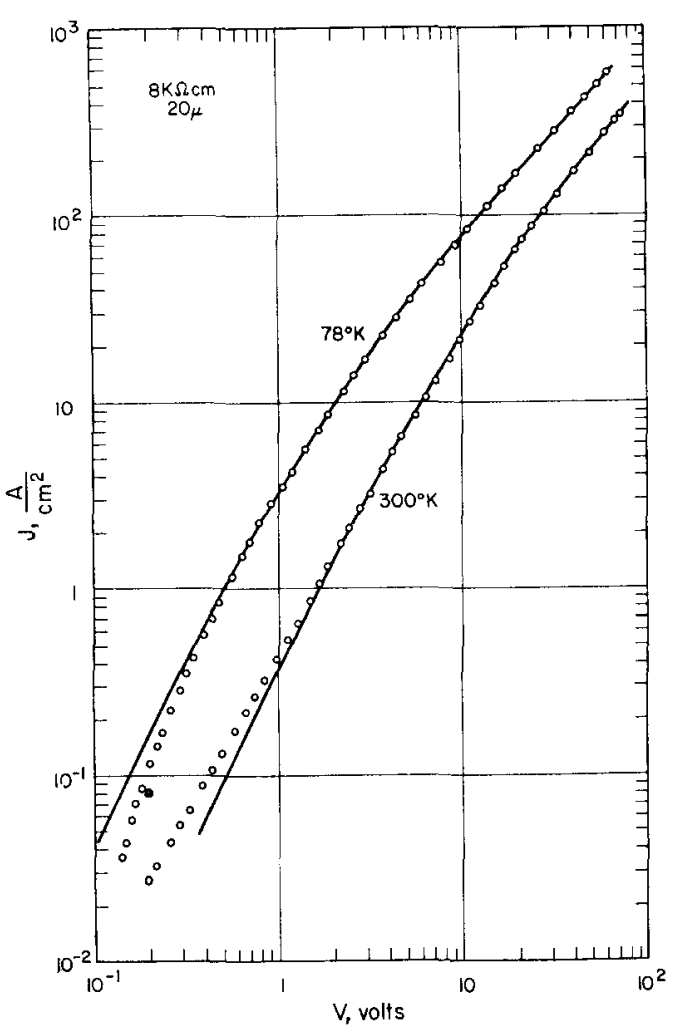

FIG. 18. The theoretical curve of Fig. 9 matches the experimental $V-I$ characteristics of thin samples remarkably well, indicating that in such cases this model may be a good approximation even at low operating points.

temperature dependence, the experimental results of the $8-\mathrm{k} \Omega \cdot \mathrm{cm}$ samples have not been used. An attempt to submit them to a similar analysis has met with partial failure. The difficulty is connected in part to the smaller number of characteristics available and the poor distribution of the base width values and in part to the absence of a clear pt range in most of these curves. An attempt to match the theoretical $V-I$ characteristic with tepid charge carriers to the experimental curves in the field limited by the boundaries 2 and 3 for the samples of $30 \mu$ and $54 \mu$ base width gave values of $E_{c}$ in the range of $1000 \mathrm{~V} / \mathrm{cm}$ to $3000 \mathrm{~V} / \mathrm{cm}$ at room temperature and between $400 \mathrm{~V} / \mathrm{cm}$ and $1300 \mathrm{~V} / \mathrm{cm}$ at $78^{\circ} \mathrm{K}$. The residual doping concentration $N$ most consistent with the corresponding asymptotes of the pt range was $1.7 \times 10^{12} \mathrm{~cm}^{-3}$. This latter value agrees moderately well with that of $2.0 \times 10^{12} \mathrm{~cm}^{-3}$ measured directly; but the former are inconclusive. An estimate of the saturation velocity can be obtained by matching the theoretical curve of Fig. 9 to the same experimental curves in their high current range. The high current asymptote then indicates about $5 \times 10^{6} \mathrm{~cm} / \mathrm{sec}$ at room temperature and $1 \times 10^{7} \mathrm{~cm} / \mathrm{sec}$ at $78^{\circ} \mathrm{K}$. Simultaneously, the low current asymptote give values of the low field mobility of about $1.8 \times 10^{3} \mathrm{~cm} / \mathrm{Vsec}$ and $7 \times 10^{3} \mathrm{~cm} / \mathrm{Vsec}$, respectively. At $78^{\circ} \mathrm{K}$, this agrees well with the results derived from the $300-\Omega \cdot \mathrm{cm}$ samples, but the values at room temperature are inconsistent. Another fact of interest is reported in Fig. 18. The $V-I$ characteristic of the $20-\mu$ sample is compared with that of Fig. 9. The two curves match over 3 decades of current. In this case, the asymptotes give about $1 \times 10^{7} \mathrm{~cm} / \mathrm{sec}$ for $v_{s}$ and $2 \times 10^{3} \mathrm{~cm}^{2} /$ Vsec for $\mu_{0}$ at room temperature. This agrees reasonably well with other data. At $78^{\circ} \mathrm{K}$, however, one finds about $1.5 \times 10^{7} \mathrm{~cm} / \mathrm{sec}$ for $v_{s}$ and $3.10^{4} \mathrm{~cm}^{2} / V$ sec for $\mu_{0}$. The value of $v_{s}$ seems a little high, but that of $\mu_{0}$ is certainly much more so when compared with the values above. Interestingly enough, though, it falls within $30 \%$ of the value extrapolated from a room-temperature mobility of $1.4 \times 10^{3} \mathrm{~cm}^{2} / \mathrm{Vsec}$ with a $T^{-2.5}$ dependence. The exact significance of these results has not been clarified, nor is it understood why the results of the $8-\mathrm{k} \Omega \cdot \mathrm{cm}$ samples should scatter as much as they do.It is certain, however, that for $8-\mathrm{k} \Omega \cdot \mathrm{cm}$ silicon and base widths of the order of $10 \mu$ the Debye length is no longer negligible. This complicates a theoretical analysis appreciably. On the other hand, the remarkable fit of Fig. 18 and the deviations in opposite directions at $78^{\circ} \mathrm{K}$ and at room temperature indicate that the model of Fig. 9 may still offer a basis for a first approximation to the characteristics in the lower left corner of the $V-I$ plane which is ignored in this work.

No breakdown has been observed in the samples presented here. There is no evidence of traps in the sclc range of the $V-I$ characteristics studied. Yet, breakdown at high voltages and trapping effects at low temperatures are known to occur. An extension of this work in these directions will be necessary to establish a connection with these phenomena.

Our acknowledgments go to A. Shumka for valuable discussions, to the U.S. Naval Ordnance Test Station, Pasadena Annex, and to the Jet Propulsion Laboratory, who have, contributed in parts to the financial support of this work. 